\title{
Les Lettres d'un voyageur. Entre fiction et autobiographie, entre quête et apprentissage
}

\section{Nigel Harkness}

\section{(2) OpenEdition}

\section{Journals}

\section{Édition électronique}

URL : http://journals.openedition.org/recherchestravaux/187

DOI : 10.4000/recherchestravaux.187

ISSN : 1969-6434

Éditeur

UGA Éditions/Université Grenoble Alpes

\section{Édition imprimée}

Date de publication : 15 avril 2007

Pagination : 95-104

ISBN : 978-2-84310-107-7

ISSN : 0151-1874

\section{Référence électronique}

Nigel Harkness, "Les Lettres d'un voyageur. Entre fiction et autobiographie, entre quête et apprentissage ", Recherches \& Travaux [En ligne], 70 | 2007, mis en ligne le 18 décembre 2013, consulté le 08 septembre 2020. URL : http://journals.openedition.org/recherchestravaux/187 ; DOI : https:// doi.org/10.4000/recherchestravaux.187 
Nigel HARKNESS

Queen's University, Belfast

\section{Les Lettres d'un voyageur}

\section{Entre fiction et autobiographie, entre quête et apprentissage}

Pour aborder les Lettres d'un voyageur, deux intertextes sandiens peuvent être privilégiés : la vie de l'auteur et les romans des années I830. Parmi ces derniers, c'est Lélia qui prédomine : la première des Lettres d'un voyageur adressée à Musset paraît dans la Revue des Deux Mondes en mai I834, quelques mois seulement après la publication de Lélia; la dernière des Lettres est publiée en novembre I836, au moment où Sand se mettait à réviser $L e ́ l i a^{1}$. La lettre IV confirme par ailleurs que ce roman était une préoccupation presque constante à l'époque de la rédaction des Lettres d'un voyageur : "Je suis dans une situation d'esprit qui ressemble tellement à celle que j'ai dépeinte, et que j'éprouvais en faisant ce livre [Lélia]» (p. 754-755; p. I40²).

Les parallèles entre ces deux textes sont nombreux : on trouve dans l'un et l'autre une structure fragmentaire, des cris de désespoir et de doute, la tentation du suicide, et un réseau symbolique opposant chaleur et froideur, corps et âme, mort et vie. En même temps, malgré les multiples identités adoptées par le personnage du narrateur au cours de ces Lettres et «le flou des voix, des moi ${ }^{3}$ » qui caractérise ce texte, il est impossible de lire les Lettres d'un voyageur sans faire des liens avec la vie de George Sand.

I. Elle annonce à Buloz en mai I836 qu'elle était «en train de faire d'importantes corrections à Lélia" (G. Sand, Correspondance, op. cit., vol. III, p. 392). Le premier extrait de ce qui allait devenir le Lélia de I839 est publié dans la Revue des Deux Mondes en juillet I836 (c'est le chapitre LVII, "Les Morts»), et un second paraît en décembre (chapitre LVIII, "Contemplation").

2. Selon la convention que nous adoptons pour ce volume: G. Sand, Lettres d'un voyageur, pages de l'édition de G. Lubin puis pages de l'édition de H. Bonnet.

3. M.-J. Hoog, "Lettres d'un voyageur, texte initiatique ", George Sand, Actes du colloque de Cerisy-la-Salle, I3-23 juillet 198I, S. Vierne (dir.), Paris, Sedes, 1983, p. I39. 
Les références autobiographiques y sont en effet nombreuses : le «je» du texte parle de sa liaison avec Musset, de son procès en séparation, de ses amis, et même de ses romans ${ }^{4}$. Autant on peut insister sur la distance entre la voix narrative et celle de l'auteur dans certains romans sandiens, autant cette distance se rétrécit dans les Lettres d'un voyageur. Néanmoins, il ne s'agit pas ici d'une autobiographie au sens strict du terme. Lorsque Sand reconnaît dans la préface de la seconde édition (I843) qu'elle s'était mise en scène dans cet ouvrage, elle insiste aussi sur sa dimension fictive, liée à la masculinité :

Que les amateurs de fiction me pardonnent un peu cependant. Dans plusieurs de ces lettres, j'ai travaillé pour eux en habillant mon triste personnage, mon pauvre moi, d'un costume qui n'était pas habituellement le sien.

Elle admet également parler «tantôt comme un écolier vagabond, tantôt comme un vieux oncle podagre, tantôt comme un jeune soldat impatient» (p. $646 ;$ p. 38). Loin de poser problème, ce brouillage des genres entre fiction et autobiographie permet de lire les Lettres d'un voyageur comme une autobiographie fictive dans laquelle la construction d'une identité littéraire et l'affirmation d'une unité entre le moi "auctorial» et cette identité fictive (" George Sand») sont des enjeux principaux 5 .

La critique a souvent considéré les Lettres d'un voyageur selon le schéma de la quête identitaire, lecture suggérée par le Voyageur lui-même quand il affirme qu'un "voyage [...] est un abrégé de la vie de l'homme» (lettre X; p. 900 ; p. 278). Marie-Jacques Hoog insiste sur le caractère initiatique de cette «quête itinérante» qui va "transformer la voyageuse [sic] en shaman, le poète en prophète» et mener à la fin à "la propagation de la foi ${ }^{6}$ ». Nicole Mozet, pour sa part, souligne le mouvement des Lettres d'un voyageur vers une unité et une identité finales car, avec l'inscription du pseudonyme "George Sand ", «est désormais acquise une fois pour toutes l'identité unificatrice de tous les narrateurs des lettres précédentes ${ }^{7}$. De ce point de vue, l'ouvrage dépeint la naissance d'un écrivain qui a trouvé non seulement sa liberté et son unité, mais aussi sa voix.

Ce texte hétérogène que Sand décrit dans sa préface comme une «œuvre

4. Voir la lettre IV : «Montrez-nous le chemin de Bernica, ou le lac de Sténio, ou les glaciers de Jacques! » (p. 747 ; p. 133).

5. L'aspect fictif de ce texte est souligné par le fait que l'organisation de l'ouvrage impose une progression factice, arrangée, qui ne respecte ni la chronologie de la rédaction, ni celle de la publication : la lettre XII fut en réalité publiée en mai I836, et devrait, si la structure avait respecté la chronologie de la publication, prendre la place de l'actuelle lettre VIII.

6. M.-J. Hoog, "Lettres d'un voyageur, texte initiatique ", op. cit., p. 137.

7. N. Mozet, "Le voyageur sandien en quête d'un lieu d'écriture ", Études françaises, vol. 24, $\mathrm{n}^{\circ}$ I, printemps 1988, George sand. Voyage et écriture, J. Goldin (dir.), p. 54. 
informe» (p. 649 ; p. 4I) reprend en effet la structure de la quête - le voyage comprend une série d'aventures et conflits (agon) qui, après une lutte centrale (pathos), mènent à un moment de découverte et d'apothéose (anagnorisis). Comme la quête, l'ouvrage est également fondé sur un constant mouvement en avant, et la reprise de certains motifs et allusions, d'une lettre à une autre, assure une téléologie poétique (voire musicale) là où une structure logique semble parfois manquer. La gondole de Catullo qui attend le Voyageur à la fin de la première lettre, va devenir dans les deux lettres suivantes son principal moyen de transport ; les ruines de la lettre III qui lui rappellent la France, assurent la transition vers la lettre IV où le Voyageur a retrouvé son pays et sa province ; la fin de la lettre VI annonce le désir de partir vers "la verte Bohême», préparant ainsi le voyage interrompu dans la lettre VII, dont les réflexions sur Lavater seront reprises dans la lettre VIII dans une analyse de la physionomie de Talleyrand (p. 856 ; p. 236) ; enfin, sur le plan artistique, les références fréquentes à la musique aboutissent à la lettre XI sur Meyerbeer, et les observations qu'on y trouve à propos de la critique annoncent le thème central de la lettre XII. Tous les maillons de cette chaîne mènent inévitablement à cette dernière lettre où la distance entre l'auteur et le personnage du Voyageur se dissout entièrement, et où Sand défend ses romans contre les attaques du critique Nisard, affirmant en même temps ce qui devrait être le souci de tout auteur, «l'éternelle infirmité humaine» (p. 942 ; p. 318). L'affirmation d'une identité littéraire permettant au Voyageur de dépasser le personnel afin de parler au nom de toute une génération, constitue ainsi l'aboutissement des quêtes identitaire et artistique contenues dans le texte.

Sur le plan autobiographique, ce schéma évolutif rappelle ce que Sand elle-même a écrit à propos de Lélia dans une lettre à Marie d'Agoult en juillet I836: «Je refais Lélia [...] le poison qui m’a rendu malade est maintenant un remède qui me guérit. Ce livre m'avait précipité dans le scepticisme ; maintenant, il m'en retire ${ }^{8}$. Si les premières Lettres d'un voyageur, qui suivent la fin de la liaison avec Musset, sont marquées par une liberté et une autonomie retrouvées, elles expriment néanmoins la souffrance et le doute, ainsi qu'une déconnexion entre l'écrivain et la société. Cette indépendance est ensuite menacée par le procès en séparation, pendant lequel les attaques personnelles se multiplient (lettre IX). Mais une fois le procès gagné, l'écrivain peut se remettre en route et retrouver une communauté d'artistes autour de Liszt et de Marie d'Agoult. Dans la lettre X, c'est l'optimisme qui domine, on y trouve un élan vers l'avenir et une foi en l'humanité. Là s'arrête plus ou

8. G. Sand, Correspondance, op. cit., vol. III, p. 474. 
moins ce qu'on pourrait appeler la trame autobiographique de l'ouvrage, car Sand ne termine pas les Lettres d'un voyageur sur ces expériences; plutôt, elle organise le volume pour que les deux dernières lettres, qui parlent de la musique, de la littérature et de la critique, sortent du domaine privé dont relèvent les autres lettres. Cette ouverture sur le domaine public s'accompagne d'un changement de destinataire : les dix premières lettres sont adressées à un "tu ", y compris la lettre VIII sur Talleyrand qui consiste en la transcription d'un dialogue avec un "tu» qui serait, d'après Georges Lubin, François Rollinat ${ }^{9}$; mais dans les deux dernières lettres, le Voyageur s'adresse à un "vous». Il s'exprime non pas comme ami, mais comme auteur, et abandonne le cadre privé des relations personnelles pour s'engager dans la sphère publique en tant que "George Sand». C'est d'ailleurs la seule fois dans l'ouvrage que ce nom paraitt ; dans d'autres lettres, quand le personnage du Voyageur est nommé, c'est toujours par un prénom, d'abord comme Zorzi dans la lettre II (p. 687; p. 76), puis comme «le petit George» dans la lettre VI (p. 785 ; p. I68), et enfin comme "George» dans la lettre X (p. 903 ; p. 280). La création et l'affirmation d'une nouvelle identité fictive et linguistique - le fait d'être devenu(e) George Sand - marque l'aboutissement de la quête identitaire et coïncide avec la projection de cette identité, en dehors du monde des rapports privés, vers un engagement public dont l'apprentissage $\mathrm{du}$ « je» masculin est la condition sine qua non.

L'intervention directe dans des débats sociaux et artistiques se fera, bien sûr, dans ses romans, mais l'espace où la voix masculine que Sand adopte ici se fait d'abord entendre, ce sont ses préfaces qui le constituent. Dans les années I830, Sand n'investissait pas totalement cet espace qui relie la fiction au monde. À partir de I833, comme Ann McCall Saint-Saëns le souligne, le prénom de l'auteur s'est figé en George sans «s» dans sa correspondance et le fait de dire «je, George Sand» en privé est alors acquis ${ }^{\circ}$. Mais dans les préfaces des années I830 - celles d'Indiana et des Romans et Nouvelles ${ }^{\text {II }}$ l'auteur évite de parler d'elle-même à la première personne, préférant faire référence à

9. La seule exception est la lettre III, la dernière des lettres de Venise, qui se conforme au modèle du récit de voyage dans la mesure où la dimension ethnographique y est dominante et où le «tu ", auquel les autres lettres vénitiennes étaient adressées, est absent.

Io. Voir A.E. McCall Saint-Saëns, De l'être en lettres, L'autobiographie épistolaire de George Sand, Amsterdam-Atlanta, Rodopi, 1996, p. 74.

II. Comme nous le signale Anna Szabó, cette dernière préface est plus souvent mentionnée comme étant celle du Secrétaire intime, alors qu'il s'agit en fait "d'un premier regard rétrospectif sur l'œuvre et en même temps une réponse aux attaques de la critique " (Préfaces de George Sand, op. cit., p. 39, note $\mathrm{n}^{\circ}$ 18). Pour ce qui est de la préface des Maîtres mosaïstes (I837), Sand s'exprime là au « je » masculin, mais la préface se situe plutôt dans le domaine du 
«l'auteur», «l'écrivain» ou «le narrateur». Dans la préface d'Indiana, elle insiste sur la jeunesse et l'inexpérience du narrateur, qualifié de «simple diseur» et de "timide ${ }^{\mathrm{i2}}$ ", caractéristiques qui le rapprochent du personnage du Voyageur dans les premières Lettres d'un voyageur. Elle n'utilise la première personne que dans un seul paragraphe au milieu de la préface où on trouve des expressions comme "ce me semble", "vous me reprocherez", " vous trouverez mauvais que je n'aie pas $^{13} »$. Mais c'est encore de manière indirecte qu'elle s'y exprime, comme si elle se sentait interpellée par un adversaire et devait y répondre. Ce n'est qu'à la fin de la préface que le «je» apparaît sur un mode plus positif: «Pensez-vous que cette vérité dégoûte les grandes âmes de l'honneur? Je pense tout le contraire ${ }^{14} »$. Encore là, il n'y a pas de fusion explicite de ce "je» avec la personne de l'auteur, et rien ne permet de dire s'il s'agit d'un "je» au masculin ou au féminin. Dans Romans et Nouvelles, on retrouve le même schéma, et la défense de ses ouvrages qu'elle y entreprend se fait également à la troisième personne : «Depuis quelques mois", écrit-elle, «les attaques dirigées contre l'auteur de Lélia ont pris un caractère tellement grossier, tellement personnel, qu'une réponse publique est devenue nécessaire ${ }^{15}$ ». Attaquée dans sa personne, on peut comprendre qu'elle hésitait à répondre publiquement en disant «je»; mais cette nécessité de se cacher derrière un personnage masculin, de répondre de façon indirecte, affaiblit sa défense de ses romans et le personnage de l'auteur y paraît impuissant à se défendre. Ce n'est qu'avec la préface du Lélia de I839, la première à suivre la publication des Lettres d'un voyageur, que l'auteur commence vraiment à parler à son public au «je» masculin : "Je me souviens de m'être complu», "cette prédilection $[\ldots]$ m'a conduit ${ }^{16} »$. Et ses remarques y prennent un caractère direct, acerbe et vigoureux, capable donc d'égaler la force, sinon l'agressivité, des attaques dirigées contre sa personne :

Quoique certaines critiques de Lélia aient revêtu un ton de déclamation et d'amertume singulières, je les ai toutes acceptées comme sincères et comme partant des cours les plus vertueux. À ce point de vue, j'ai eu lieu de me réjouir et de penser que j'avais mal jugé les hommes de mon temps, en les contemplant à travers un douloureux scepticisme. Tant d'indignation attestait sans doute de la part des journalistes la plus haute moralité, jointe à la plus religieuse philanthro$\mathrm{pie}^{\mathrm{I}}$.

privé, ayant la forme d'une lettre adressée à «Maurice D... » par « [s] on vieux père » (G. Sand, Vies d'artistes, Paris, Presses de la Cité, 1992, p. 37).

I2. G. Sand, Indiana, édition de B. Didier, Paris, Gallimard «Folio », I984, p. 38.

13. Ibid., p. 39.

I4. Ibid., p. 4I.

15. G. Sand, Le Secrétaire intime, Grenoble, Éditions de l'Aurore, I99I, p. I9.

I6. G. Sand, Lélia, édition de P. Reboul, Paris, Garnier, I960, p. 350. 
La rhétorique prend ici une force de contestation et de persuasion qui manquait dans les préfaces écrites à la troisième personne. Sand laisse ainsi derrière elle le flou des voix qui caractérisait les préfaces antérieures, pour écrire à partir d'une nouvelle solidarité avec l'identité fictive que représentait son pseudonyme. On peut en effet lire les Lettres d'un voyageur comme la mise en abyme d'un processus qui aboutit à une affirmation de soi et à une autonomie à la fois personnelle et littéraire, dont les conséquences dépassent le monde de la fiction. C'est donc, comme le suggère Nicole Mozet, le récit de la "réconciliation avec elle-même d'une personnalité foncièrement double (Aurore/George), longtemps contrainte à une vie éclatée et même masquée ${ }^{18}$ ».

Pourtant, bien que le caractère initiatique des Lettres d'un voyageur semble clair, la notion de quête n'est pas sans poser problème. Dans Histoire de ma vie, Sand affirme qu'elle voulait faire dans les Lettres d'un voyageur «le propre roman de ma vie», mais sans en être «le personnage réel», et que cette stratégie lui permettait d'étendre le point du vue du narrateur «à une expérience du malheur que je n'avais pas, que je ne pouvais pas avoir ${ }^{19}$ ». Le personnage $\mathrm{du}$ Voyageur serait, toujours d'après Histoire de ma vie, "une sorte de fiction, un personnage convenu, masculin comme mon pseudonyme», "un héros de roman $^{20}$ », un «moi fantastique ${ }^{21} »$. La notion de quête privilégie un mouvement téléologique, mais Sand souligne les expériences dont est constituée cette exploration identitaire. Par ailleurs, il s'agit ici d'une quête par procuration dans laquelle la masculinité établit une distance entre le moi de l'auteur et celui du Voyageur. Malgré l'affirmation par l'écrivain de son identité littéraire, l'unité entre le moi féminin et un «George Sand» masculin n'est pas totale. La lettre XII est marquée par des hésitations, et le Voyageur s'y décrit comme un «talent incomplet qui n'a pas encore atteint sa virilité, et qui peutêtre, hélas! ne sortira jamais de son enfance» (p. 938 ; p. 314). Si la quête identitaire conclut sur la fusion du moi féminin avec une identité publique masculine, cette lettre ne met pas un point final au processus de développement. En fait, elle annonce la naissance d'un écrivain pour qui le difficile apprentissage de la masculinité ne fait que commencer.

La recherche identitaire dépeinte dans les Lettres d'un voyageur est profondément marquée par un voyage vers le masculin, comme en témoigne la

17. Ibid., p. 349 .

I8. N. Mozet, "Signé "le voyageur" : George Sand et l'invention de l'artiste », Romantisme, $\mathrm{n}^{\circ} 55, \mathrm{I}^{\mathrm{er}}$ trim., 1987, p. 23.

19. G. Sand, Histoire de ma vie, édition de G. Lubin, op. cit., vol. II, p. 299.

20. Ibid., vol. I, p. 7 .

2I. Ibid., vol. II, p. 299. 
réécriture au masculin des lettres IV et $\mathrm{IX}^{22}$. Mais le texte exprime aussi une prise de conscience des enjeux de la masculinité qui ressort de façon particulièrement nette dans une des oppositions structurantes de l'ouvrage : l'eau et la pierre ${ }^{23}$. Une étude de cette opposition permet de souligner les oscillations entre masculin et féminin dans le texte et de mettre en valeur la difficulté de devenir George Sand.

La pierre est presque toujours associée à la masculinité dans les Lettres d'un voyageur. Comme l'indique Yvette Bozon-Scalzitti, «de la pierre, l'homme a la solidité et la stabilité24 », mais ces caractéristiques prennent ici des connotations négatives dans la mesure où elles se rapprochent dans l'esprit du Voyageur d'une attitude de détachement. Composantes majeures de l'axe vertical du texte, les rochers et les montagnes permettent de dominer la nature et indiquent une position de maitrise virile vis-à-vis du monde. Dans la première lettre, le Voyageur se réveille sur une cime des Alpes et, de cette position, il a l'impression que les plaines qui se déroulent devant lui sont comme "une conquête épuisée, comme un espace déjà franchi». Animé d'une force "active et violente», "orgueilleuse», il ressent la tentation d'une "éternelle solitude», mais doit résister, car une voix intérieure lui dit que ce n'est pas la solution qu'il cherche : "C'est donc là où tu en es ? [...] eh bien! marche, avance, apprends» (p. $674 ;$ p. 63). Alors que la pierre fait partie de cet axe vertical qui doit être rejeté, l'eau est l'élément clé de l'axe horizontal. Contre la pierre, symbole de détachement et de domination, l'eau signifie l'égalité et la fraternité. D'après le Voyageur, l'une des grandes qualités de Venise - cette ville de marbre et d'eau - est l'égalité des manières qui y règne.

22. Elles faisaient originellement une seule lettre, écrite au féminin, publiée dans la Revue des Deux Mondes en 1836.

23. Voir Y. Bozon-Scalzitti, «La pierre, l'eau, le sable : l'écriture sandienne dans les Lettres d'un voyageur", Nineteenth-Century French Studies, vol. XXI, n 3-4, 1993, p. 34I. Dans cet article, Yvette Bozon-Scalzitti se penche sur cette opposition entre pierre et eau dans le cadre d'une étude de la "problématique identité masculine du voyageur " (p. 34I). Sa démarche critique est fortement influencée par des perspectives psychanalytiques dans lesquelles les rapports de l'auteur avec sa mère et son père sont privilégiés. Elle fait ainsi une équivalence entre "pétrification funèbre » associée au marbre, et «maîtrise masculine ", à travers une "identification au père disparu » (p. 342). Son travail a néanmoins le grand mérite de souligner les dissonances et les déchirements de ce texte pluriel.

24. Seul Éverard (Michel de Bourges) est épargné. Dans la lettre VI le narrateur loue la force et l'énergie de son ami : "Ce serait en vain que le monde tomberait en poussière autour de toi ; tu te ferais marbre alors, et, comme Atlas, tu porterais la terre sur tes épaules inébranlables » (p. 795; p. 177). Le fait d'être de marbre accentue sa force et fait de lui un héros. Si le marbre prend ici un sens positif, c'est qu'il n'est plus synonyme de détachement et d'insensibilité. 
Le fait que tout le monde doive aller sur l'eau, et par conséquent l'absence de chevaux et de voitures, font que : "Personne ne crotte et n'écrase son semblable. Il n'y a point là l'humiliation de passer à pied auprès d'un carrosse" (lettre III ; p. 729 ; p. II6). La verticalité des rapports est donc abolie, et le bateau devient le moyen de transport privilégié dans ces lettres, car il permet de rassembler sur un pied d'égalité des amis et des connaissances, sans égard pour les différences de sexe, de classe ou de nationalité.

Si le Voyageur privilégie l'eau, c'est aussi pour s'opposer aux injustices d'une société pétrifiée qui fait «sentir ses angles de marbre et ses herses de fer "(lettre X ; p. 903 ; p. 280). Sur le plan symbolique, l'eau attaque les anciennes certitudes, comme le dit le moine arménien que le Voyageur rencontre à Venise : «les doctrines nouvelles s'infiltrent peu à peu dans l'antique vérité, comme l'eau dans le marbre» (lettre III ; p. 7I8 ; p. I05). L'eau devient donc une force déstabilisante dont ce moine a peur ; en effet, ceux qui sont attachés aux structures de la société actuelle rejettent la mobilité, l'évolution, le renouvellement. Le narrateur, par contre, se décrit comme «un bon marcheur et un rude casseur de pierres» (lettre V; p. 774 ; p. 158), mettant ainsi en valeur deux caractéristiques associées à l'eau : la mobilité et le refus de toute fixité. C'est pourquoi le Voyageur a tellement peur, pendant cet épisode de la première lettre, quand il voit son reflet dans une eau «transparent [e] et immobile comme un bloc d'émeraude» (p. 667 ; p. 56). Cette fixité est aliénante, et le reste du texte est caractérisé par la fuite du Voyageur devant la pétrification, synonyme de détachement et d'isolation, c'est-à-dire aussi d'insensibilité et de dureté. Dans la lettre VII, le Voyageur exprime sa peur d'être devenu lui-même dur et insensible comme la pierre :

J'ai épaissi mon écorce, j'ai durci la peau de mes pieds sur les pierres de tous les chemins, et je me rappelle avec étonnement ces jours de ma jeunesse où la moindre inquiétude, où la moindre espérance me crispaient comme une sensitive. Pourquoi suis-je devenu un rocher? (P. 843 ; p. 223.)

Et il parle avec admiration des organisations supérieures qui ne ressentent pas cette séparation avec le monde matériel : «En elles l'enveloppe terrestre est si éthérée, si diaphane, si impressionnable, que l'esprit qui les anime semble voir et pénétrer à travers la matière qui enveloppe ou compose le monde extérieur. Leur fibre est si tendre et si déliée que tout ce qui échappe aux sens grossiers des autres hommes la fait vibrer, comme la moindre brise émeut et fait frémir les cordes d'une harpe éolique» (p. 843 ; p. 222-223). À l'impassibilité et l'indifférence de l'être en pierre, le Voyageur oppose la perméabilité et l'ouverture de l'être supérieur.

Dans ce texte, le va-et-vient entre la pierre et l'eau, le masculin et le fémi- 
nin, exprime une hantise permanente : la crainte, en adoptant une identité masculine, même fictive, de devenir insensible, de se détacher des autres. Comment éviter, en effet, que la solidité d'une identité masculine n'enferme le moi dans l'individualisme et l'égoïsme ? La solution que Sand envisage ici semble prendre la forme d'une descente symbolique sur terre qui permet de canaliser le personnel dans l'universel. Plus tôt, dans la lettre VII, le Voyageur avait déjà dit n'être plus attiré par "d'ardentes aspirations vers le ciel", et parle de "ces cimes sublimes d'où l'on descend pour n'y plus remonter" (p. 823 ; p. 204). Dans la lettre IX, il passe de «la hauteur culminante», de «la dernière pierre de la colline» (p. 875; p. 254) au «revers du rocher dans le plus profond du ravin» (p. 876 ; p. 255). En laissant ainsi derrière lui cet axe de la verticalité si dominant dans les premières lettres, la transcendance - cet élan vers l'idéal, cette fuite hors du monde matériel - est transformée en un mouvement vers les autres, un engagement social ; l'artiste ne vivra plus retranché de ses semblables, mais interviendra à côté d'eux et exprimera sa solidarité avec l'humanité et ses souffrances. En jouant sur l'homonymie, on peut affirmer que le Voyageur descend des hauteurs pour s'affirmer comme auteur. Je lirais l'affirmation à la fin de la lettre XII dans ce contexte. La formule " George Sand et compagnie» reprend peut-être, sur un ton de dédain et d'ironie, les propos de Nisard, exprimant la solidarité de l'auteur avec les écrivains et artistes ayant adopté les mêmes positions esthétiques et politiques qu'elle. Mais il signifie également un rejet du détachement et une affinité avec ses semblables, écrivains ou non, qui installe l'auteur au sein d'une communauté à la fois littéraire et sociale. L'identité du Voyageur se solidifie au cours de ce texte, mais la conclusion exprime la détermination que George Sand ne sera jamais un être en pierre, insensible et distant.

Dans les Lettres d'un voyageur, il s'agit pour Sand moins de faire de la fiction à partir d'expériences autobiographiques, que de réussir l'intégration de sa personne avec un pseudonyme à travers lequel elle intervient dans la sphère publique en parlant à la première personne au masculin. Elle se crée en s'écrivant. Ayant adopté au cours de ce texte de multiples identités - celle d'un jeune voyageur, $d^{\prime}$ ' un poète, c'est-à-dire une femmelette» (lettre VI ; p. 806 ; p. I87), ou d'un "pauvre diseur de métaphores» (lettre VI ; p. 78I ; p. I64) la voix narrative se solidifie dans les dernières lettres, et prend le nom George Sand. C'est cette voix masculine que nous allons entendre, à la première personne, dans les préfaces qui suivront les Lettres d'un voyageur. Pourtant, si le narrateur des Lettres d'un voyageur réussit au terme de son voyage une réconciliation avec la masculinité obligée de la sphère publique, une étude des processus de cette affirmation identitaire montre que ce Voyageur reste néanmoins conscient des dangers d'une identification totale avec la masculinité, 
associée au détachement et à l'isolement. Les Lettres d'un voyageur finissent donc non pas sur un «je, George Sand", mais sur un «nous», "George Sand et compagnie ${ }^{25}$ " (p. 942 ; p. 318). Dans ce texte caractérisé par l'hétérogénéité, le singulier fait vite la place au pluriel, la solidité et la séparation sont remplacées par la mobilité et la perméabilité du moi, et à la linéarité de la quête se substituent les tensions d'un apprentissage qui est loin d'être terminé.

25. Là où Yvette Bozon-Scalzitti voit une «occultation du féminin » («La pierre, l'eau, le sable : l'écriture sandienne dans les Lettres d'un voyageur", op. cit., p. 340), je verrais plutôt une dualité masculin-féminin, sans pourtant que les tensions et oscillations entre ces deux pôles soient résolues dans une harmonie androgyne. 